\title{
Infrared observatory prepares for lift-off
}

\section{Tony Reichhardt, Washington}

The last of NASA's 'great observatories' - a set of space telescopes conceived by the agency almost a quarter-century ago - is finally ready for launch.

If all goes to plan, the Space Infrared Telescope Facility (SIRTF) will go into orbit on a Delta rocket from Cape Canaveral, Florida, on 18 April - having survived years of delays and doubts that, its designers say, have ultimately resulted in a more capable observatory.

It is the fourth in a series of powerful NASA space telescopes covering different parts of the electromagnetic spectrum, after the Hubble Space Telescope, the Compton Gamma Ray Observatory and the Chandra $\mathrm{X}$-ray Observatory.

SIRTF's infrared detectors are especially suited to studying cool objects, such as planets in the act of formation and brown dwarfs — bodies that lack the mass to ignite nuclear fusion and become stars. It will detect radiation that passes through dust that blocks observations at other wavelengths, as well as signals from very distant, fast-receding galaxies whose visible light is Doppler-shifted into the infrared spectrum.

The space observatory's 85-centimetre mirror is small by ground-based standards. But because of the sensitivity of its detectors and their placement in the cold vacuum of space, infrared observations that would require exposures of several hours from the ground "will happen in seconds", says Michael Werner, the SIRTF project scientist at NASA's Jet Propulsion Laboratory in Pasadena, California.
For Werner and others, it has been a long, tortuous trip to the launch pad. He began working on SIRTF in 1977. When NASA asked astronomers to propose instruments for the observatory six years later, the plan was to carry it into orbit inside a space shuttle. By the mid-1990s, though, the estimated cost of SIRTF - which had by then been reconfigured as a separately launched satellite had ballooned to $\$ 2.2$ billion, far more than NASA could afford. The observatory was scaled back in size, and its observing range narrowed to wavelengths of 3-180 $\mu \mathrm{m}$. It ended up costing $\$ 670$ million to build, and will cost a further $\$ 500$ million to launch and operate.

In some ways, the design emerged stronger. The observatory needs to be cooled by liquid helium to stop it emitting infrared noise that will interfere with its observations. Mission planners found a means of cutting the amount of helium needed by a factor of ten - instead of following a conventional orbit, the spacecraft will drift behind Earth as it circles the Sun, where the environment is cooler. That idea, plus modifications to the cooling system, should keep SIRTF running for five years or more. The previous best orbiting infrared telescope, Europe's Infrared Space Observatory, operated for only 28 months before its helium ran out in 1998.

While SIRTF's launch date kept slipping in the 1990s, infrared detectors kept improving in sensitivity. And because the spacecraft itself will be so cold, and therefore low in infrared emissions, the new detectors will be able to exploit their sensitivity to the full, says

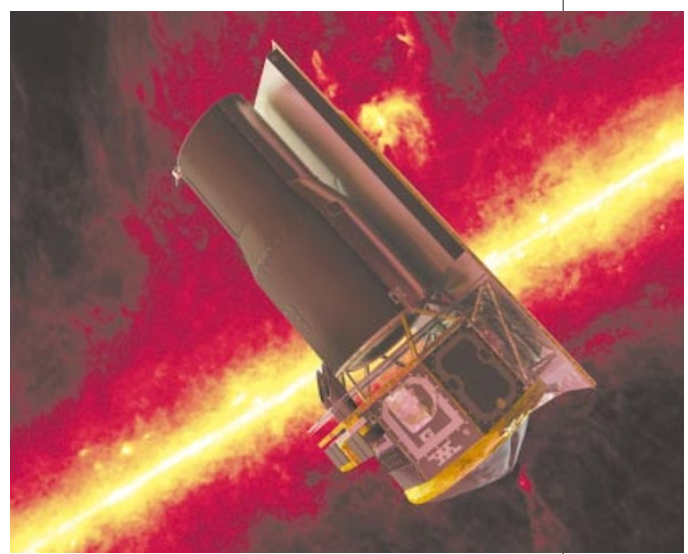

The last of NASA's great observatories, SIRTF will provide rapid data on cool objects in the Universe.

George Rieke of the University of Arizona at Tucson, principal investigator for one of the observatory's three instruments.

Like the other three great observatories, SIRTF will be used by large numbers of astronomers around the world. It is expected to view some 20,000 targets annually, thanks to a faster pace of observation than other space telescopes: whereas Hubble looks at about ten objects a day and Chandra just three, SIRTF should rack up 50 or 60.

But for many SIRTF scientists, getting to the launch pad is a triumph in itself. Rieke recalled at a recent press conference that his team had applied to participate in the project on Friday 13 May 1983. "Somehow," he says, "we have overcome the bad aura."

http://sirtf.caltech.edu

\section{High-voltage shock sparks fusion from X-ray crush}

\section{Geoff Brumfiel, Philadelphia}

A small burst of nuclear fusion has been induced for the first time using $\mathrm{X}$-rays generated by a huge burst of electricity, according to US researchers.

Scientists from Sandia National Laboratory in New Mexico told the American Physical Society's meeting in Philadelphia on 5 April that the results were generated by the lab's Z-machine in late March. "This is the first observation of fusion from an electrical source," says Sandia physicist Ray Leeper.

Fusion occurs when two small nuclei usually of hydrogen or one of its isotopes, deuterium or tritium - fuse together to form a larger one. Physicists try to reproduce the phenomenon, which powers the stars, using one of two techniques: magnetic confinement, which holds a plasma in place magnetically and heats it; or inertial confinement, which uses $\mathrm{X}$-rays to crush a small target so rapidly that fusion takes place.

The Z-machine attempts the latter, using energetic X-rays to crush a small sample of deuterium to form helium. The $\mathrm{X}$-rays are generated by releasing a massive electrical power surge through a small cylinder of 360 tungsten filaments, which surrounds a 2-mm pellet filled with the deuterium. At the flip of a switch, scientists release a 40-terawatt pulse just 75 nanoseconds long through the filaments, vaporizing the wires instantly, and emitting X-rays that crush the pellet.

The Z-machine has been running since September 1996, but last month's experiment confirmed that emissions of neutrons - a by-product of the fusion reaction - had been detected.

The result could strengthen the case for a larger Z-machine - which Sandia has wanted to build for years, at a cost of several hundred million dollars. It may also help with research at the National Ignition Facility, an experiment under construction at the Lawrence Livermore National Laboratory in California, which will use the world's most powerful laser to achieve inertial confinement fusion.

The fusion energy generated in the experiment is estimated to be 4 millijoules - about a billionth of the electricity put in - so the technique has some way to go in terms of efficiency.

But Per Peterson, chair of the nuclear engineering department at the University of California, Berkeley, says it could one day be possible to scale up something like the Z-machine to create an affordable fusion power plant. "Pulsed power has the potential to be by far the cheapest approach" to fusion power, he says. 